Physics

Physics Research Publications

\title{
Out-of-Plane Momentum and Symmetry-Dependent Energy Gap of the Pnictide Ba0.6K0.4Fe2As2 Superconductor Revealed by Angle-Resolved Photoemission Spectroscopy
}

Y. Zhang, L. X. Yang, F. Chen, B. Zhou, X. F. Wang, X. H. Chen, M. Arita,

K. Shimada, H. Namatame, M. Taniguchi, J. P. Hu, B. P. Xie, and D. L. Feng 


\title{
Out-of-Plane Momentum and Symmetry-Dependent Energy Gap of the Pnictide $\mathrm{Ba}_{0.6} \mathrm{~K}_{0.4} \mathrm{Fe}_{2} \mathrm{As}_{2}$ Superconductor Revealed by Angle-Resolved Photoemission Spectroscopy
}

\author{
Y. Zhang, ${ }^{1}$ L. X. Yang, ${ }^{1}$ F. Chen, ${ }^{1}$ B. Zhou, ${ }^{1}$ X. F. Wang, ${ }^{2}$ X. H. Chen, ${ }^{2}$ M. Arita, ${ }^{3}$ K. Shimada,${ }^{3}$ H. Namatame, ${ }^{3}$ \\ M. Taniguchi, ${ }^{3}$ J.P. Hu, ${ }^{4}$ B.P. Xie, ${ }^{1}$ and D. L. Feng ${ }^{1, *}$ \\ ${ }^{1}$ State Key Laboratory of Surface Physics, Department of Physics, and Advanced Materials Laboratory, \\ Fudan University, Shanghai 200433, People's Republic of China \\ ${ }^{2}$ Department of Physics, University of science and technology of China, Hefei, Anhui 230027, People's Republic of China \\ ${ }^{3}$ Hiroshima Synchrotron Radiation Center and Graduate School of Science, Hiroshima University, Hiroshima 739-8526, Japan \\ ${ }^{4}$ Department of Physics, Purdue University, West Lafayette, Indiana 47907, USA
}

(Received 20 June 2010; published 8 September 2010)

\begin{abstract}
The three-dimensional band structure and superconducting gap of $\mathrm{Ba}_{0.6} \mathrm{~K}_{0.4} \mathrm{Fe}_{2} \mathrm{As}_{2}$ are studied with angle-resolved photoemission spectroscopy. In contrast with previous results, we have identified three holelike Fermi surface sheets near the zone center with sizable out-of-plane or $k_{z}$ dispersion. The superconducting gap on certain Fermi surface sheets shows significant $k_{z}$ dependence. Moreover, the superconducting gap sizes are different at the same Fermi momentum for two bands with different spatial symmetries (one odd, one even). Our results further reveal the three-dimensional and orbital-dependent structure of the superconducting gap in iron pnictides, which facilitates the understanding of momentumintegrated measurements and provides a distinct test for theories.
\end{abstract}

DOI: 10.1103/PhysRevLett.105.117003

PACS numbers: 74.25.Jb, 71.20.-b, 74.70.-b, 79.60.-i

The discovery of high- $T_{c}$ superconductivity in iron pnictides has ignited extensive studies. However, the pairing symmetry is still not settled. Most of the present theories propose an $s_{ \pm}$nodeless order parameter that is isotropic on one Fermi pocket and changes sign between the hole and electron pockets $[1,2]$. However, there is conflicting experimental evidence for the presence of nodes and nodeless superconducting gaps $[3,4]$. Furthermore, strongly anisotropic and multiple gap behavior has been deduced based on transport measurements [5-7]. One possible cause of these controversies is the multiband nature of iron-based superconductors that all the five $\mathrm{Fe} 3 d$ orbitals participate in the low-lying electronic structure, giving hole pockets at the zone center and electron pockets at the zone corner $[8,9]$. The importance of Fermi surface topology and its orbital character has been pointed out by recent theories $[10,11]$. It is proposed that the presence of a node is determined by the number of bands which cross the Fermi level and that there is a strong anisotropy and amplitude variation of the superconducting gaps on different Fermi surface sheets. Moreover, various physical properties of iron-based superconductors are more three dimensional (3D) than the cuprates. For example, the isotropy of the upper critical field has been found in $\mathrm{Ba}_{1-x} \mathrm{~K}_{x} \mathrm{Fe}_{2} \mathrm{As}_{2}$ [12].

Previous angle-resolved photoemission spectroscopy (ARPES) studies have shown isotropic nodeless gaps on all the Fermi surface sheets in $\mathrm{Ba}_{1-x} \mathrm{~K}_{x} \mathrm{Fe}_{2} \mathrm{As}_{2}$ [13-15], $\mathrm{BaFe}_{2-y} \mathrm{Co}_{y} \mathrm{As}_{2}$ [16], and $\mathrm{Fe}_{1.03} \mathrm{Te}_{0.7} \mathrm{Se}_{0.3}$ [17]. The most representative and detailed data to date were taken on the optimally doped $\mathrm{Ba}_{1-x} \mathrm{~K}_{x} \mathrm{Fe}_{2} \mathrm{As}_{2}$ samples [13-15,18-20], where two hole pockets near the zone center were observed. The superconducting gap on the inner hole pocket is found to be larger than that on the outer pocket, which is consistent with the $s_{ \pm}$pairing symmetry with a gap function proportional to $\left|\cos k_{x} \cos k_{y}\right|$. However, there are still many issues to be resolved. For example, calculations predicted three hole bands at the zone center rather than two [9-11]. The relationship between orbital characters and superconducting gaps is yet to be established. Furthermore, band calculation suggested that the 3D character of the electronic structure is important for the magnetism and superconductivity in iron-based superconductors [21]. However, due to the limited photon energies used in previous ARPES studies, the superconducting gaps along the out-of-plane momentum $\left(k_{z}\right)$ direction have not been exposed.

In this Letter, we have studied the $k_{z}$ dependence of the superconducting gap in high-quality $\mathrm{Ba}_{0.6} \mathrm{~K}_{0.4} \mathrm{Fe}_{2} \mathrm{As}_{2}$ single crystals with ARPES. We found that the Fermi surface near the zone center $\Gamma$ actually contains three hole pockets instead of two as in previous studies. Significant $k_{z}$ dependence of the superconducting gap is discovered on one of the bands. Particularly, we found that at the same momentum, bands with different orbital symmetries could exhibit very different gap sizes. Our results provide a more global picture of the gap, which would help the construction of microscopic models of the iron-based superconductors.

High-quality $\mathrm{Ba}_{0.6} \mathrm{~K}_{0.4} \mathrm{Fe}_{2} \mathrm{As}_{2}\left(T_{c}=38 \mathrm{~K}\right)$ single crystals were synthesized by the self-flux method [22] with a superconducting transition width of $0.5 \mathrm{~K}$. Data were taken with various photon energies at beam line 5-4 of the Stanford Synchrotron Radiation Laboratory (SSRL), and with the $21.2 \mathrm{eV}$ helium- $I \alpha$ line of a discharge lamp in 
mixed polarization geometry. Polarization-dependent data were taken at beam line 1 of the Hiroshima synchrotron radiation center (HSRC). Two polarization geometries $\left(E_{p}, E_{s}\right)$ were achieved by rotating the experimental chamber. All the data were taken with Scienta electron analyzers; the overall energy resolution is $15 \mathrm{meV}$ at $\mathrm{HSRC}$ or $7 \mathrm{meV}$ at SSRL, and angular resolution is $0.3^{\circ}$. The samples were cleaved in situ, and measured under ultrahigh vacuum of $5 \times 10^{-11}$ Torr.

Since the superconducting gap is almost isotropic around the Fermi surface cross section of certain $k_{z}[14,15]$, we focus on the $(0,0)-(\pi, \pi)$ high symmetry cut for simplicity. The photon energy dependent data are shown in Fig. 1. Only two bands could be resolved in $25 \mathrm{eV}$ [Fig. 1(a)], forming two hole pockets as reported in previous studies. However, by changing the photon energy, we could observe an additional band $(\beta)$ moving outwards in 29.5 and $31 \mathrm{eV}$ [Figs. 1(b) and 1(c)]. The Fermi momenta $\left(k_{F}\right)$ of these three bands are determined by peak positions in momentum distribution curves (MDCs) at the Fermi energy $\left(E_{F}\right)$ [23] in Fig. 1(d). Taking the inner potential of $15 \mathrm{eV}$ [24] to calculate the $k_{z}$ 's of different photon energies, our data cover half of the Brillouin zone along the $k_{z}$ direction from $\Gamma$ with $\sim 21 \mathrm{eV}$ photons to $Z$ with $\sim 31 \mathrm{eV}$ photons [Fig. 1(e)]. While $\alpha$ and $\gamma$ show little $k_{z}$ dispersion, $\beta$ is almost degenerate with $\alpha$ near $\Gamma$, but moves outward significantly near $Z$. Since the photon energies applied in

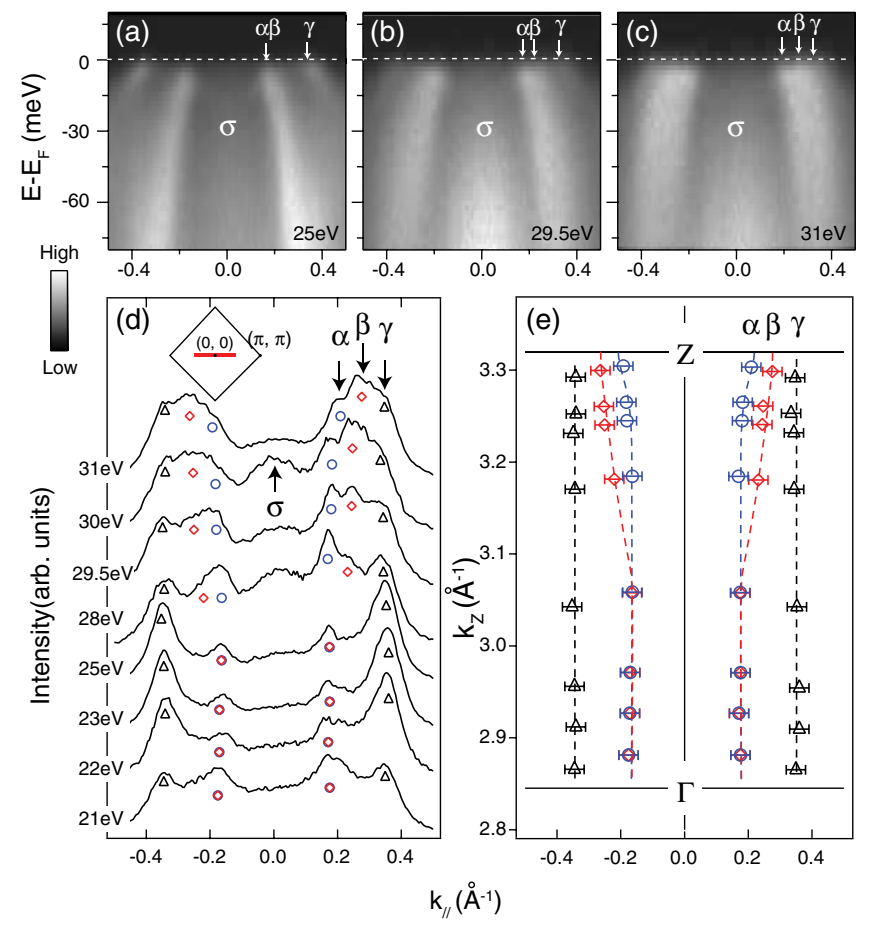

FIG. 1 (color online). (a)-(c) Photoemission data of $\mathrm{Ba}_{0.6} \mathrm{~K}_{0.4} \mathrm{Fe}_{2} \mathrm{As}_{2}$ along the $(0,0)-(\pi, \pi)$ direction taken with 25, 29.5, and $31 \mathrm{eV}$, respectively. (d) The MDCs at $E_{F}$ taken with different photon energies are stacked. The inset shows the projection of the cuts in the 2D Brillouin zone. (e) The $k_{F}$ 's determined from the MDCs in (d). Data were taken at $10 \mathrm{~K}$. most previous ARPES studies could just sample the $\Gamma$ region, $\alpha$ and $\beta$ could not be distinguished [13-15,18$20,25,26]$. Therefore, three hole pockets reported here with distinct $k_{z}$ dispersions naturally resolve the previous inconsistency with band calculations [10,11], which predicted three hole pockets around the zone center.

To further reveal the orbital characters of these three bands, we have conducted polarization-dependent ARPES experiments. The polarization geometry $E_{p}\left(E_{s}\right)$ could be achieved with the polarization direction parallel (perpendicular) to the mirror plane defined by the sample normal and the $(0,0)-(\pi, \pi)$ direction. The orbitals of even (odd) symmetry with respect to the mirror plane could be observed in $E_{p}\left(E_{s}\right)$ geometry [27]. The polarizationdependent data around $Z$ are shown in Figs. 2(a)-2(d). $\beta$ could be observed in $E_{p}$ geometry; thus, it is of an even orbital character. Oppositely, $\alpha$ only shows up in the $E_{s}$ geometry, which suggests an odd orbital character. $\gamma$ is mostly of odd orbital character [Figs. 2(c) and 2(d)], but there is also some trace of $\gamma$ in $E_{p}$ geometry due to possible orbital mixing. The MDCs at $E_{F}$ of different polarization geometries around $Z$ are compared in Fig. 2(e). The peak positions in $E_{p}$ and $E_{s}$ geometry are consistent with the data taken in mixed polarization geometry. Since the different orbital symmetries of $\alpha$ and $\beta$ should not change along the $k_{z}$ direction [21], it enables us to separate them with polarization-dependent experiment around $\Gamma$. The MDC peaks of $\alpha$ and $\beta$ show up at the same momentum in Fig. 2(j). This further proves that the $k_{F}$ 's of the $\alpha$ and $\beta$ bands almost degenerate around $\Gamma$, but separate from each
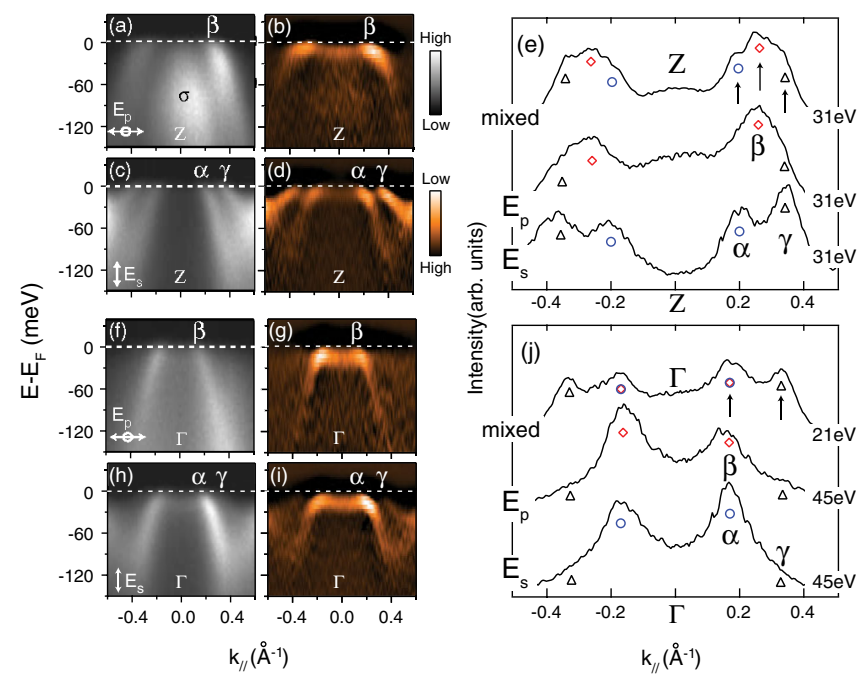

FIG. 2 (color online). (a) Photoemission intensity near $Z$ along the $(0,0)-(\pi, \pi)$ direction taken in the $E_{p}$ geometry with $31 \mathrm{eV}$ photons. (b) The second derivative with respect to energy of data in (a). Panels (c) and (d) are the same as (a) and (b), respectively, but taken in the $E_{s}$ geometry. (e) The MDCs at $E_{F}$ with mixed, $E_{p}$, and $E_{s}$ polarization geometries near $Z$. Panels (f)-(i) are the same as (a)-(d), respectively, but taken with $45 \mathrm{eV}$ photons to reach the $\Gamma$ point. (j) The MDCs at $E_{F}$ with mixed, $E_{p}$, and $E_{s}$ polarization geometries near $\Gamma$. Data were taken at $40 \mathrm{~K}$. 
other around $Z$. Note that the intensity of $\gamma$ is very weak in the data taken with $45 \mathrm{eV}$ photons, possibly due to the matrix element effects of this particular photon energy.

The two odd and one even bands observed here qualitatively agree with the band calculations, where only three orbitals $\left(d_{x z}, d_{y z}\right.$, and $\left.d_{x y}\right)$ contribute to the low energy electronic structure around $\Gamma[10,11] . \beta$ is $d_{x z}$ orbital due to its even symmetry. $\gamma$ shows little $k_{z}$ dispersion, which is most likely the $d_{x y}$ orbital with two-dimensional (2D) character. $\alpha$ is thus assigned to the $d_{y z}$ orbital, which is predicted to degenerate with the $d_{x z}$ orbital at $\Gamma$ by theory. Note that there are some broad spectral weights $(\sigma)$ at $(0,0)$, which is possibly due to the contribution of $d_{z^{2}}$ bands below $E_{F}$ or the incoherent spectral weight scattered from other states [Figs. 1 and 2(a)]; they are thus ignored in the following discussions.

The data in the superconducting state near $\Gamma$ are shown in Figs. 3(a) and 3(b). The $\gamma$ band shows simple Bogoliubov dispersion with a gap about $4 \mathrm{meV}$. Most notably, at the $k_{F}$ 's of the $\alpha$ and $\beta$ bands, the energy distribution curves (EDCs) exhibit a complex structure with two peaks [Figs. 3(g) and 3(h)]. The peak positions could be tracked towards $\Gamma$ with Bogoliubov dispersion of two energy scales (7 and $11.5 \mathrm{meV}$ ) as shown in Fig. 3(e). The energy scale of $11.5 \mathrm{meV}$ is consistent with the gap size observed in previous studies [14]. Moreover, the clear bending-over behavior and small peak width clearly reproduce the
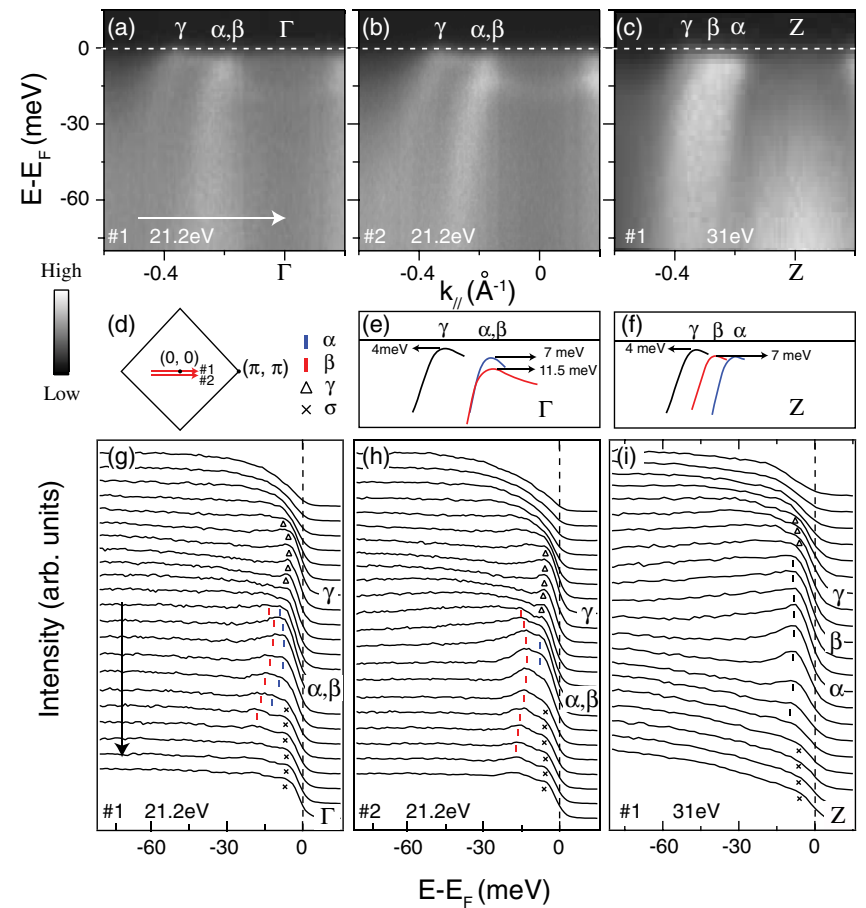

FIG. 3 (color online). (a),(b) Photoemission intensities taken with $21.2 \mathrm{eV}$ along two momentum cuts as indicated in (d). (c) Same as (a), but taken near $Z$ with $31 \mathrm{eV}$ photon energy. Panels (e) and (f) are illustrations of the bands in (a) and (c), respectively. (g)-(i) EDCs of the data in (a)-(c), respectively. Data were taken at $10 \mathrm{~K}$. properties of a superconducting peak. Furthermore, the peak at $7 \mathrm{meV}$ should not be the bent-over feature of $\gamma$, as one can track the $\gamma$ band by the triangles in Figs. 3(g) and 3(h). It quickly bends over to high binding energies and loses its weight significantly. Therefore, the most natural explanation of our results is that $\alpha$ and $\beta$ have different superconducting gaps at the same momentum. On the other hand, while these three bands could be clearly separated around $Z$ in Fig. 3(c), only single peaks could be observed at the $k_{F}$ 's of $\alpha$ and $\beta$ with a superconducting gap of $7 \mathrm{meV}$ [Fig. 3(i)].

In the five-band model, the Fermi surface sheets of ironbased superconductors consist of sections with different orbital characters $[8,11]$. The multiorbital interactions could form strong gap anisotropy on the Fermi surface sheets. Therefore, the observation of two energy scales of $\alpha$ and $\beta$ could be related to their orbital nature, since they show opposite orbital symmetries at the same Fermi momentum. That is, the pairing strengths could be strongly determined by the orbital symmetries. The next question is which band provides a larger gap around $\Gamma$. Considering there is a significant change from 11.5 to $7 \mathrm{meV}$ for one of the gaps along the $k_{z}$ direction, plus the strong $k_{z}$ dependence of $\beta$, it is reasonable to assume that $\beta$ contributes to the larger gap of $11.5 \mathrm{meV}$ around $\Gamma$. We could not completely exclude the possibility of $\alpha$ at this stage, but this assumption would only affect the gap assignment around $\Gamma$, and would not affect the observed $k_{z}$ dependence of the superconducting gaps discussed below. Polarizationdependent experiments with high resolution in the superconducting state are needed to clarify this point. We leave this for future studies.

The symmetrized EDCs at the $k_{F}$ 's of the $\alpha, \beta$, and $\gamma$ bands are summarized in Figs. 4(a)-4(c). The two-peak behavior could only be resolved around $\Gamma$. The superconducting gap values are determined by fitting with the common phenomenological superconducting spectral function as illustrated in Fig. 4(a) [28]. The $k_{z}$ dependence of superconducting gaps is shown in Fig. 4(e). The gap sizes of $\alpha$ and $\gamma$ are about 7 and $4 \mathrm{meV}$, respectively, and the gap sizes of $\beta$ show strong $k_{z}$ dependence from 11.5 to $7 \mathrm{meV}$. In previous ARPES studies, the gap sizes around $\Gamma$ could be fitted well with $\Delta_{0}\left|\cos k_{x} \cos k_{y}\right|$ [14-17]. Therefore, the superconducting gap should decrease away from the $(0,0)$ point. We plot the superconducting gap versus $\left|\cos k_{x} \cos k_{y}\right|$ in Fig. 4(g). The $\alpha$ and $\gamma$ bands could be well fitted in this relation with $\Delta_{0} \approx 8 \mathrm{meV}$, as shown by the straight line in Fig. 4(g). However, the large deviation of the $\beta$ band (highlighted by the shaded region) indicates that the $k_{z}$ dependence of the gap sizes of $\beta$ could not be explained by the change of in-plane Fermi surface size. Therefore, the $k_{z}$ contribution must be included to describe the gap function of the $\beta$ band. With the simple formula of $\Delta_{0 \beta}\left|\cos k_{x} \cos k_{y}\right|\left(1+A \cos k_{z}\right)$, one can fit the gap of $\beta$ reasonably well with $\Delta_{0 \beta} \approx 11.2 \mathrm{meV}$ and $A \approx 0.24$ as shown by the solid line in Fig. 4(e). 

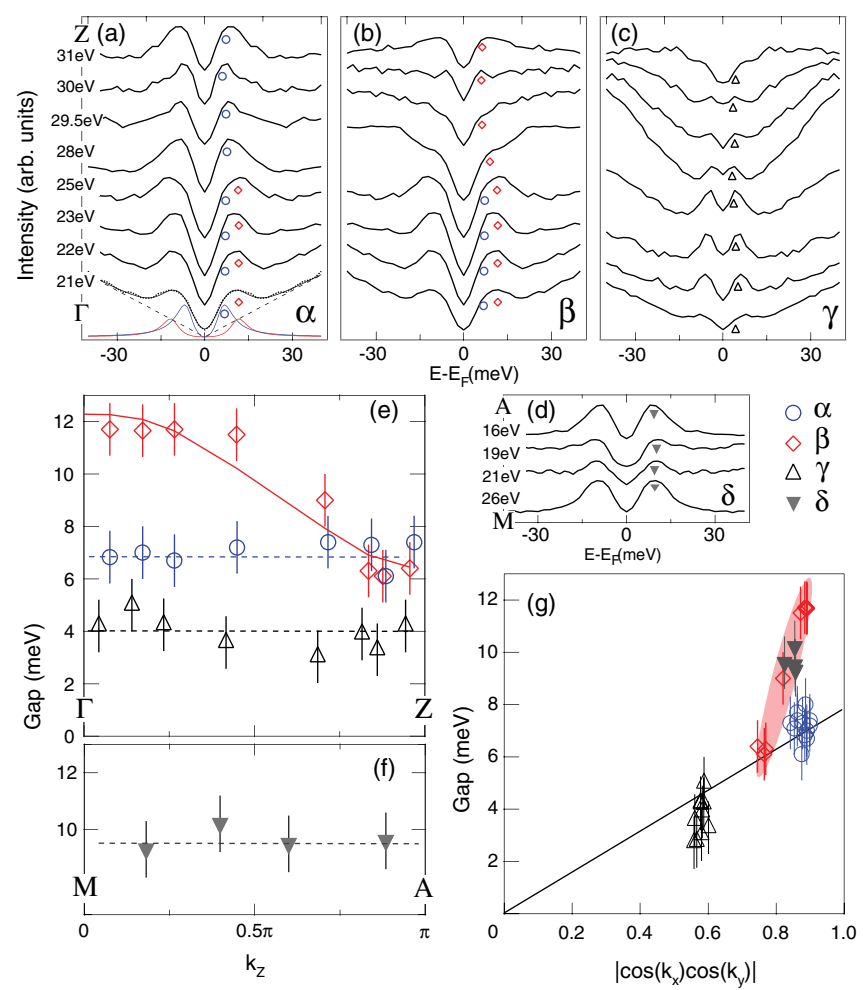

FIG. 4 (color online). (a)-(c) Symmetrized EDCs at the $k_{F}$ 's of three bands taken with different photon energies. The dashed line is the background used in the fitting process. (d) Symmetrized EDCs at the momentum $0.2 \AA^{-1}$ from $(\pi, \pi)$, which is the $k_{F}$ of electron pocket $(\delta)$ at the zone corner [14]. (e),(f) $k_{z}$ dependence of the superconducting gaps around the zone center and corner, respectively. (g) Gaps vs $\left|\cos k_{x} \cos k_{y}\right|$.

The $k_{z}$-dependent superconducting gaps discovered here could not be explained in 2D models, which are used to construct the pairing mechanism in most of the previous theories $[10,11]$. In contrast to cuprates, the iron-based superconductors show many 3D characters, e.g., the small anisotropy of the upper critical field [12,29], and 3D spin fluctuations in parent and doped compounds [30-32]. Our results further emphasize the $3 \mathrm{D}$ character of the superconductivity, where the out-of-plane pairing channels should be considered. Consistently, a substantial $k_{z}$ dependence of the superconducting order parameter has been found in a recent 3D band model that is constructed to calculate the spin fluctuations and the pairing function [21].

The superconducting gap size is proposed to be related to the nesting condition of Fermi surface sheets between $\Gamma$ and $M$ in previous ARPES studies $[14,16]$. The Fermi surface sizes near the zone corner show weak $k_{z}$ dependence $[33,34]$. In that case, the change of the Fermi surface size of $\beta$ along the $k_{z}$ direction could break the nesting condition, and thus significantly change the superconducting gaps. However, it could not explain the different gap sizes of $\alpha$ and $\beta$, since they have almost the same Fermi surface size around $\Gamma$. Moreover, the $k_{z}$ dependence of gaps near the zone corner is not as obvious, as shown in Figs. 4(d) and 4(f), and the gap sizes also deviate from the fitting of the $\left|\cos k_{x} \cos k_{y}\right|$ relation in Fig. 4(g). Therefore, the pairing between $\Gamma$ and $M$ could not be related to just simple nesting of the Fermi surface. The orbital character and the 3D electronic structure should be taken into account.

To summarize, the superconducting gap in $\mathrm{Ba}_{0.6} \mathrm{~K}_{0.4} \mathrm{Fe}_{2} \mathrm{As}_{2}$ shows strong out-of-plane and orbital symmetry dependence, which could be related to the $3 \mathrm{D}$ electronic structure and multiorbital nature of iron-based superconductors. Our results have set up a more comprehensive picture of the superconducting gap in this compound.

We gratefully acknowledge the experimental support by Dr. D. H. Lu and Dr. R. G. Moore at at SSRL. This work was supported by the NSFC, MOE, MOST (National Basic Research Program No. 2006CB921300), and STCSM of China. SSRL is operated by the U.S. DOE Office of Basic Energy Science.

\section{*dlfeng@fudan.edu.cn}

[1] I. I. Mazin et al., Phys. Rev. Lett. 101, 057003 (2008).

[2] Kangjun Seo, B. A. Bernevig, and Jiangping Hu, Phys. Rev. Lett. 101, 206404 (2008).

[3] Clifford W. Hicks et al., Phys. Rev. Lett. 103, 127003 (2009).

[4] T. Y. Chen et al., Nature (London) 453, 1224 (2008).

[5] K. Gofryk et al., New J. Phys. 12, 023006 (2010).

[6] T. J. Williams et al., Phys. Rev. B 80, 094501 (2009).

[7] K. W. Kim et al., Phys. Rev. B 81, 214508 (2010).

[8] K. Kuroki et al., Phys. Rev. Lett. 101, 087004 (2008).

[9] S. Graser et al., New J. Phys. 11, 025016 (2009).

[10] F. Wang, H. Zhai, and D. H. Lee, Phys. Rev. B 81, 184512 (2010).

[11] Ronny Thomale et al., arXiv:1002.3599.

[12] H. Q. Yuan et al., Nature (London) 457, 565 (2009).

[13] H. Ding et al., Europhys. Lett. 83, 47001 (2008).

[14] K. Nakayama et al., Europhys. Lett. 85, 67002 (2009).

[15] L. Wray et al., Phys. Rev. B 78, 184508 (2008).

[16] K. Terashima et al., Proc. Natl. Acad. Sci. U.S.A. 106, 7330 (2009).

[17] K. Nakayama et al., arXiv:0907.0763.

[18] V. B. Zabolotnyy et al., Nature (London) 457, 569 (2009).

[19] P. Richard et al., Phys. Rev. Lett. 102, 047003 (2009).

[20] C. Liu et al., Phys. Rev. Lett. 101, 177005 (2008).

[21] S. Graser et al., Phys. Rev. B 81, 214503 (2010).

[22] X. F. Wang et al., Phys. Rev. Lett. 102, 117005 (2009).

[23] A. Kaminski et al., Phys. Rev. Lett. 86, 1070 (2001).

[24] P. Vilmercati et al., Phys. Rev. B 79, 220503(R) (2009).

[25] P. Richard et al., Phys. Rev. Lett. 104, 137001 (2010).

[26] L. X. Yang et al., Phys. Rev. Lett. 102, 107002 (2009).

[27] Y. Zhang et al., arXiv:0904.4022.

[28] M. R. Norman et al., Phys. Rev. B 57, R11 093 (1998).

[29] M. Fang et al., Phys. Rev. B 81, 020509(R) (2010).

[30] J. Zhao et al., Phys. Rev. Lett. 101, 167203 (2008).

[31] R. J. McQueeney et al., Phys. Rev. Lett. 101, 227205 (2008).

[32] Songxue Chi et al., Phys. Rev. Lett. 102, 107006 (2009).

[33] Walid Malaeb et al., J. Phys. Soc. Jpn. 78, 123706 (2009).

[34] C. Liu et al., Phys. Rev. Lett. 102, 167004 (2009). 